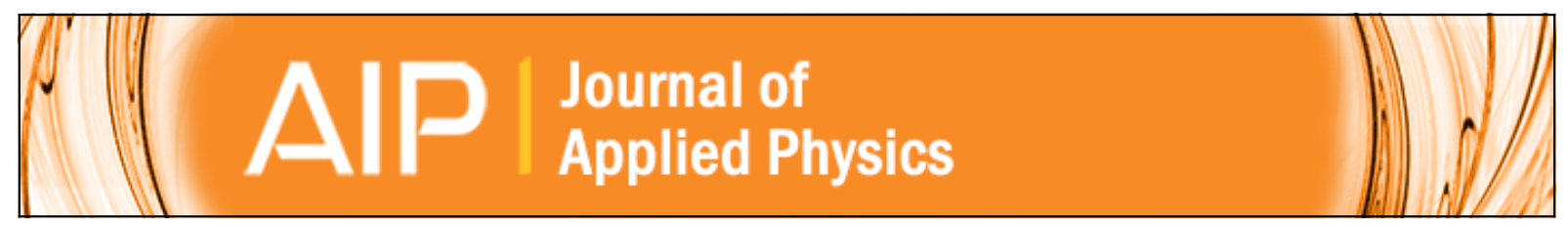

\title{
Characterization of atomic force microscope probes at low temperatures
}

Alexandra Radenovic, Eva Bystrenova, Laurent Libioulle, Francesco Valle, George T. Shubeita, Sandor Kasas, and Giovanni Dietler

Citation: Journal of Applied Physics 94, 4210 (2003); doi: 10.1063/1.1604952

View online: http://dx.doi.org/10.1063/1.1604952

View Table of Contents: http://scitation.aip.org/content/aip/journal/jap/94/6?ver=pdfcov

Published by the AIP Publishing

\section{Articles you may be interested in}

In-situ scanning electron microscopy and atomic force microscopy Young's modulus determination of indium oxide microrods for micromechanical resonator applications

Appl. Phys. Lett. 104, 161909 (2014); 10.1063/1.4872461

Cantilever temperature characterization in low temperature vacuum atomic force microscope

Rev. Sci. Instrum. 77, 043704 (2006); 10.1063/1.2188417

Dynamical properties of the $\mathrm{Q}$-controlled atomic force microscope

Appl. Phys. Lett. 85, 3232 (2004); 10.1063/1.1785863

Sub-attonewton force detection at millikelvin temperatures

Appl. Phys. Lett. 79, 3358 (2001); 10.1063/1.1418256

Low-temperature atomic force microscope using piezoresistive cantilevers

Rev. Sci. Instrum. 70, 3369 (1999); 10.1063/1.1149945

\section{A|P| Journal of}

Journal of Applied Physics is pleased to announce André Anders as its new Editor-in-Chief 


\title{
Characterization of atomic force microscope probes at low temperatures
}

\author{
Alexandra Radenovic, Eva Bystrenova, ${ }^{\text {a) }}$ Laurent Libioulle, Francesco Valle, and George \\ T. Shubeitab) \\ Institute of Condensed Matter Physics, University of Lausanne, CH-1015 Lausanne, Switzerland \\ Sandor Kasas \\ Laboratory of Cellular Neurobiology, FSV-EPFL, CH-1015 Lausanne, Switzerland \\ and Institute of Cellular Biology and Morphology, University of Lausanne, CH-1015 Lausanne, Switzerland \\ Giovanni Dietler \\ Institute of Condensed Matter Physics, University of Lausanne, CH-1015 Lausanne, Switzerland
}

(Received 30 April 2003; accepted 8 July 2003)

\begin{abstract}
Different types of atomic force microscopy (AFM) probes were characterized under ultrahigh vacuum conditions and at low temperatures. Properties of AFM probes, such as the resonance frequency, the spring constant and quality factor of cantilevers, depend on temperature. A typical shift in the resonance frequency as a function of temperature was observed for all kinds of cantilevers studied. This was related to the change in temperature of Young's modulus of the cantilever material. Moreover, force-distance curves acquired at low temperatures and on different substrates, elucidate the importance of the hydrophobicity of the sample surface and that of the tips for lowering adhesion forces. Finally, all of the probes were imaged in a scanning electron microscope as a function of the temperature. A bending of the coated cantilever at low temperatures was observed, which explains the peculiar force-distance curves. As a consequence, the use of uncoated cantilevers for low-temperature applications is recommended. (C) 2003 American Institute of Physics. [DOI: 10.1063/1.1604952]
\end{abstract}

\section{INTRODUCTION}

Atomic force microscopy ${ }^{1}$ (AFM) has become an important tool for studying surfaces at the atomic level. Its ability to measure vertical and lateral deflections with subnanometer resolution and subnanonewton force sensitivity makes AFM powerful in investigating properties of surfaces and of different complex systems on surfaces. The accuracy of such measurements relies primarily on the physical properties of the cantilever used as a force sensor.

In AFM, the tip of a flexible force sensing cantilever is raster scanned over the sample surface. Forces acting between the tip and the sample cause deflections of the cantilever which are detected and displayed as an image. In contact mode AFM, the scanning tip is maintained in contact with the sample surface with an interaction force as small as $1-100 \mathrm{nN}$ to avoid damage. Atomic resolution images of conductive and nonconductive surfaces, as well as molecular resolution images of organic and biological materials, have been achieved in this mode. In the case of operating the microscope in dynamic mode, the force sensor could then be fixed on a quartz tuning fork. ${ }^{2,3}$ At low temperature and under an ultrahigh vacuum, this could make the design of the microscope simpler than in our laser beam deflection setup. Since, on biological samples, the friction force and some specific biochemical interactions are also valuable informa-

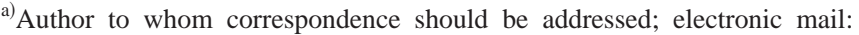
Eva.Bystrenova@ipmc.unil.ch

${ }^{b}$ Current address: Department of Developmental and Cell Biology, University of California, Irvine, Ca 92697. tion, contact mode with the standard cantilever still remains a very interesting method to image the surface. In this context, the cantilever is chosen to be stiff enough to reduce vibration noise to an acceptable level, but should still have a low spring constant $\left(10^{-2}-10 \mathrm{~N} / \mathrm{m}\right)$ to be adequately sensitive. Other crucial properties of high performance AFM levers include high resonance frequency $(>10 \mathrm{kHz})$, high mechanical quality factor (100-1000), high lateral stiffness, short lever length, and a sharp tip. ${ }^{4}$ When the AFM is operated in contact mode, the resonance frequency of the cantilever changes significantly, since the end of the cantilever is no longer free to vibrate and quality factor is of less importance due to the strong damping.

Commercial cantilevers are microfabricated from silicon or silicon-nitride $\left(\mathrm{Si}_{3} \mathrm{~N}_{4}\right)$ by photolithographic and vapor deposition methods. Unfortunately, these techniques result in variations of the dimensions of the cantilever, which in turn lead to a considerable variation in their elastic constants. Moreover, a thin layer of metal $(\mathrm{Au}, \mathrm{Al}$, and $\mathrm{Au} / \mathrm{Cr}$ ) is usually deposited onto the cantilever to increase its reflectivity when deflections are detected optically. Adding this metallic layer changes the weight and stiffness of the cantilever and, consequently, its resonance frequency.

Since most AFM studies require the properties of the cantilever to be known precisely, many methods were developed to measure the relevant properties of the specific cantilevers used. In the present article, such methods are extended for use at low temperatures and under ultrahigh vacuum conditions. Other approaches to characterizing cantilevers at low temperatures are also presented. 
TABLE I. Characterization of the three types of commercial cantilevers used in this study.

\begin{tabular}{|c|c|c|c|c|c|c|c|c|c|c|c|}
\hline Type & Material & Coating & $\begin{array}{l}\text { Length } \\
(\mu \mathrm{m})\end{array}$ & $\begin{array}{c}\text { Thickness } \\
(\mathrm{nm})\end{array}$ & $\begin{array}{l}\text { Width } \\
(\mu \mathrm{m})\end{array}$ & $\begin{array}{l}\text { Spring constant } \\
(\mathrm{N} / \mathrm{m})\end{array}$ & $\begin{array}{l}\text { Resonant frequency } \\
\qquad(\mathrm{kHz})\end{array}$ & Tip shape & $\begin{array}{l}\text { Tip height } \\
\qquad(\mu \mathrm{m})\end{array}$ & $\begin{array}{l}\text { Tip radius } \\
(\mathrm{nm})\end{array}$ & Producer \\
\hline I & $\mathrm{Si}_{3} \mathrm{~N}_{4}$ & $\begin{array}{c}\mathrm{AuCr} \\
\text { both sides }\end{array}$ & 200 & 400 & 27 & 0.02 & 11 & Pyramidal & 2.9 & 20 & $\begin{array}{l}\text { Olympus Optical } \\
\text { Co. Ltd. }\end{array}$ \\
\hline II & $\mathrm{Si}_{3} \mathrm{~N}_{4}$ & $\begin{array}{c}\text { AuCr } \\
\text { both sides }\end{array}$ & 100 & 400 & 13 & 0.08 & 34 & Pyramidal & 2.9 & 20 & $\begin{array}{l}\text { Olympus Optical } \\
\text { Co. Ltd. }\end{array}$ \\
\hline III & Silicon & No & 290 & 1000 & 40 & 0.12 & 15 & Conical & 15 & 10 & NT-MDT Co. \\
\hline
\end{tabular}

\section{EXPERIMENTAL METHODS AND RESULTS}

Measurements in the present work were performed using an atomic force microscope operating at low temperatures (cryoatomic force microscope) under ultrahigh vacuum $\left(10^{-7}-10^{-9}\right.$ mbar $)$ using the laser beam deflection method ${ }^{5}$ to measure the bending of the cantilevers. A detailed description of the instrument was reported previously. ${ }^{6}$ For measurement of the cantilever oscillation resonance spectra, a spectrum analyzer (Stanford Research System model SR780) was used.

Measurements were performed for the three different types of triangular cantilevers for contact mode AFM listed in Table I. We were particularly interested in the behavior of very soft cantilevers since they have higher force sensitivity and are thus more suitable for studying the biological samples in which we are interested.

\section{A. Cantilever resonance spectra measurements}

The accuracy of the spring constant is usually the limiting factor in the precision of a force measurement. Many methods have been used to compute or measure the spring constant of cantilevers. The most straightforward method is to measure the dimensions of the cantilever and compute the theoretical spring constant. Measurement inaccuracies of this technique-especially in the thickness result in large spring constant errors. Measuring the resonance frequency ${ }^{7}$ provides another estimation of the thickness, thus improving the accuracy of this technique.

Alternatively, beads or particles of known masses can be added to the end of the beam, while measuring the resulting resonance shifts. ${ }^{8}$ This method can be accurate only if the masses are accurately known and their location on the cantilever beam is well determined. Micromanipulating $10 \mu \mathrm{m}$ diameter beads is a time consuming task, and is likely to result in breaking the cantilever.

A different approach makes use of the intrinsic thermal vibration of the cantilever. Every mass-spring system, coupled to a thermal bath, undergoes thermal fluctuations with an expectation value proportional to the temperature and inversely proportional to the spring constant. According to the equipartition theorem of classical statistical mechanics, each degree of freedom of a simple harmonic oscillator carries a thermal energy $k_{B} T / 2$ determined only by the temperature. The spring constant can then be obtained by measuring the thermally induced fluctuations and the temperature. ${ }^{9}$ The major challenge with this method is in getting an accurate measure of the minute thermomechanical motion over a broad frequency range.
In this work, we applied nondestructive procedures ${ }^{7,9}$ for calibrating cantilevers at different temperatures with the aim to evaluate their applicability in AFM experiments. The exact value of Young's modulus is required for calculating the force constant from the measurements. Bulk values of Young's modulus can differ by $20 \%$, depending on manufacturing procedures. Furthermore, Young's modulus changes with temperature.

The dependence of Young's modulus on temperature, $T$, is given by the theory of lattice dynamics in terms of a set of parameters specifying the interatomic forces including anharmonic terms. Wachtman et al. ${ }^{10}$ confirmed a $T^{4}$ dependence for temperatures ranging from 0 to $300 \mathrm{~K}$ and a linear dependence for higher temperatures. Consequently, since the spring constant depends on Young's modulus, it is important to utilize the method by which it is possible to measure this dependence.

Figure 1 shows the thermal power spectra for the three types of cantilevers (listed in the Table I) under a vacuum and at different temperatures (listed in Table II). The temperature dependence of frequency for each of the cantilevers is shown in the inset of Fig. 1. Resonance frequency values in air and at room temperature were measured to be 4 $\pm 0.5 \%$ different from the corresponding value in a vacuum. This is in agreement with measurements reported elsewhere. $^{7,11}$

Every spectrum is an average of 20 spectra of 800 points. A Lorentzian function is fitted to the resonance peak. Values of the force constant of the cantilever are calculated at each temperature by integrating this curve. The spring constant, $k_{1}$, is calculated using the following expression ${ }^{9}$

$$
k_{1}=\frac{k_{B} \cdot T}{P}
$$

where $P$ is the area of the power spectrum of the thermal fluctuations, $k_{B}$ is the Boltzman's constant, and $T$ is the temperature. As is shown in Fig. 1 and Table II, the resonance frequency increases with decreasing temperature. From the width of resonance peaks $\Delta f$, the quality factor $Q$ $\sim f_{\exp } / \Delta f$ was calculated ${ }^{12}$ and is presented in Table II. The quality factor increases approximately three times at $82 \mathrm{~K}$ compared to the room temperature.

The spring constant can otherwise be written as a function of the geometrical and mechanical parameters of the cantilever, both of which can be easily measured or calculated as: ${ }^{7}$

$$
k_{2}=C \cdot \rho^{3 / 2} \cdot Y^{-1 / 2} \cdot f_{0}^{3},
$$




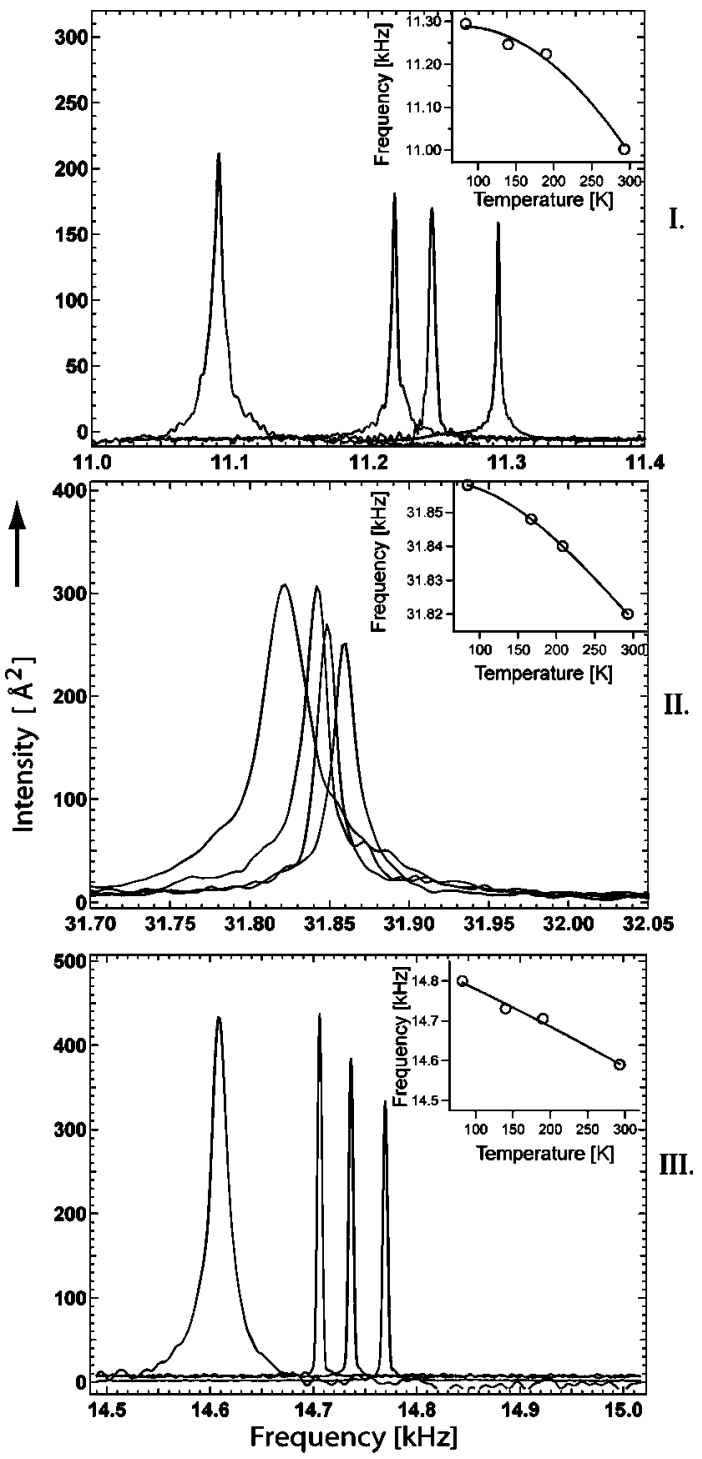

FIG. 1. The thermal noise spectra acquired at different temperatures for cantilevers of types I, II, and III. Insets show the temperature dependence of the resonance frequency.

where $C$ is geometrical factor, $Y$ is Young's modulus, $\rho$ is the density of the material of the cantilever, and $f_{0}$ is the resonance frequency. Subsequently, this method can be applied to the different temperatures just by rescaling. As shown in Table II, the biggest change of the spring constant is observed for cantilevers of type I.

Since the change in density of the material of the cantilever is negligible, we can conclude that the frequency shift is due to a change in Young's modulus and/or to a change of the form of the cantilever induced by the temperature change.

\section{B. Force-distance curve measurements}

The knowledge of the forces acting between the tip and the sample is the basis for any quantitative analysis of AFM measurements. A force-distance curve is a compact way of representing this interaction and enables better interpretation of the images acquired as well as better regulation of the scanning tip.
TABLE II. Spring constants and resonance frequencies measured at different temperatures. The quality factors calculated from the shape of resonance frequency peaks at different temperatures (see Ref. 11).

\begin{tabular}{rcccr}
\hline \hline $\begin{array}{c}\text { Temperature } \\
(\mathrm{K})\end{array}$ & $\begin{array}{c}\text { Resonant } \\
\text { frequency } \\
(\mathrm{kHz})\end{array}$ & $\begin{array}{c}\text { Spring } \\
\text { constant } k_{1} \\
(\mathrm{~N} / \mathrm{m})\end{array}$ & $\begin{array}{c}\text { Spring } \\
\text { constant } k_{2} \\
(\mathrm{~N} / \mathrm{m})\end{array}$ & $\begin{array}{c}\text { Quality } \\
\text { factor }\end{array}$ \\
\hline & & Type I & & \\
82 & 11.294 & 0.019 & 0.017 & 17740 \\
140 & 11.246 & 0.018 & 0.016 & 11776 \\
190 & 11.224 & 0.014 & 0.016 & 11753 \\
295 & 11.002 & 0.012 & 0.015 & 5760 \\
& & Type II & & \\
82 & 31.858 & 0.09 & 0.088 & 15397 \\
167 & 31.848 & 0.088 & 0.088 & 13340 \\
208 & 31.840 & 0.082 & 0.088 & 12503 \\
295 & 31.820 & 0.079 & 0.088 & 7140 \\
& & & & \\
82 & 14.800 & 0.142 & 0.115 & 15498 \\
140 & 14.730 & 0.139 & 0.114 & 15425 \\
190 & 14.705 & 0.124 & 0.110 & 13199 \\
295 & 14.590 & 0.117 & 0.110 & 4583 \\
\hline \hline
\end{tabular}

Force-distance curves were recorded using a LABVIEW program (National Instruments). A 16-bit precision analog/ digital card form National Instruments (NI PCI-MIO-16XE10) was used for both output and input. The custom-made program made it possible to record the force curves at the desired rate.

For many substrates used for biological imaging, such as mica ${ }^{13}$ and graphite, ${ }^{14}$ a large adhesion force, $F_{A}$, between the probe and the surface exists. This force is often many orders of magnitude larger than the van der Waals force and strongly pulls the probe toward the specimen surface. The adhesion force is characterized as the force required separating the tip from the surface. ${ }^{15}$

In our measurements, both freshly cleaved mica and graphite were used. Mica is known to carry some negative surface charges after cleavage. ${ }^{13}$ Because the surface charge can induce an opposite charge in the gold/chromium coating of the cantilever, this can result in a large attractive force. This can be confirmed by the fact, that when a cantilever is approaching the substrate surface, the force reaches large negative values before the tip engagement (Fig. 2-column I for $82 \mathrm{~K}$ ). Proper grounding of the specimen may lower the adhesion force. Graphite, on the other hand, is conductive and the surface charge can be totally shielded if the specimen is connected properly to the ground. The long-range interaction can be totally eliminated. ${ }^{16}$

Figure 2 shows typical force-distance curves for cantilevers used in this study (columns I-III) at different temperatures $(82 \mathrm{~K}, 190 \mathrm{~K}$, and $295 \mathrm{~K})$. Combining the results from force-distance curves with the measurements of the spring constants gives us enough information to determine the applied forces for each temperature (see inset in every graph of Fig. 2). For the large adhesion, one could lower the set point by pulling up the cantilever to reduce the force exerted on the sample. ${ }^{17}$ However, it is clear from the inset of Fig. 2, the forces used are small and fall in the range of some $100 \mathrm{pN}$. 


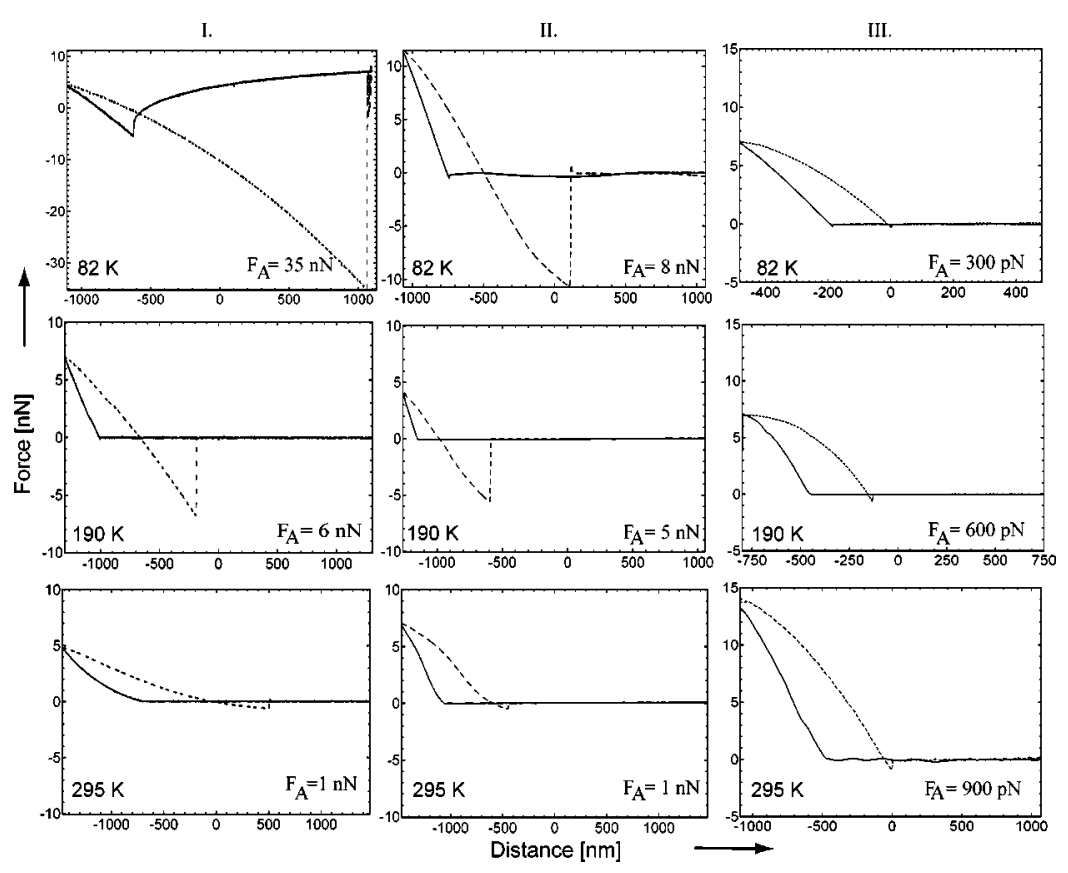

FIG. 2. The temperature dependence of force-distance curves. Column I (type I cantilever) and column II (type II cantilever): Coated cantilevers on mica. Column III (type III cantilever): Uncoated cantilever on graphite. Values inserted in graphs are average values of adhesion forces $F_{A}$. A large adhesion force is observed at low temperature while using coated cantilevers due to bad grounding of the specimen and hydrophilicity of mica, which was suppressed when using hydrophobic conductive graphite. The large adhesion observed for the long coated cantilever (column I) at $82 \mathrm{~K}$ is partially due to cantilever bending (as confirmed by cryoSEM measurement).

The origin of the adhesion force has been attributed to the presence of a water layer on the surface. Working with biological samples implies the presence of water even if the samples are dried prior to measurements (the same is valid for substrates as mica and graphite). At low temperatures, this water will form a thin layer of ice covering the sample, reducing resolution, and probably leading to the formation of a quasiliquid layer between sample and the tip. ${ }^{18,19} \mathrm{We}$ are currently trying to avoid the presence of water in our system by choosing hydrophobic substrates and tips as described next.

\section{Scanning electron microscopy measurements at low temperatures}

In order to gain better insight into the behavior of cantilevers at low temperatures, we imaged them using a scanning electron microscope operating at low temperatures (108-295 $\mathrm{K})$. Micrographs of cantilevers were recorded at different temperatures [see Figs. 3(a), 3(d), and 3(e)]. Experiments were performed using a scanning electron microscope Philips XL-30 SFEG operating at $5 \mathrm{kV}$ equipped with a field emission gun and coupled with a cryotransfer Gatan Alto 2500. A special sample holder was designed for the cantilever to be placed between two plates in order to ensure good thermal contact. Surprisingly, a bending of approximately $6^{\circ}$ is visible at $108 \mathrm{~K}$ in Fig. 3(a), even though the cantilevers (types I and II) were coated from both sides to prevent bimetallic behavior induced by the difference between the thermal expansion coefficients of the gold/chromium film and that of the cantilever.

The bending of the cantilevers was modeled using finite element analysis. The finite element analysis was performed
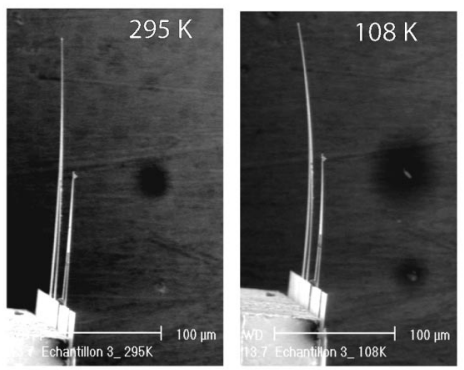

(a)

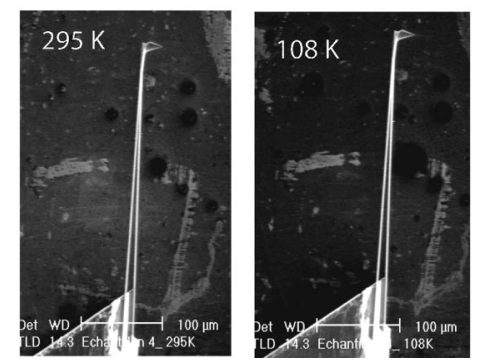

(b)

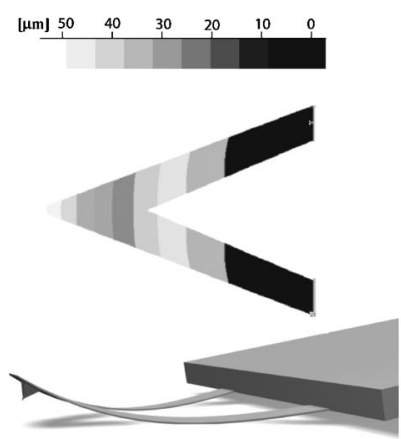

(c)

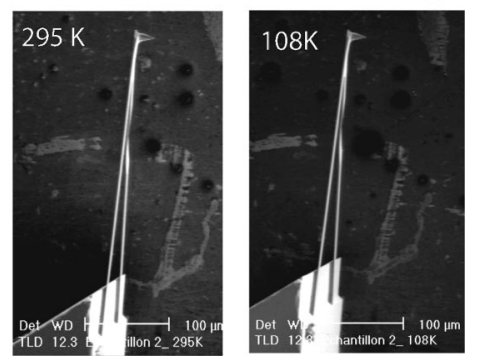

FIG. 3. (a) Micrographs of coated cantilevers, types I and II, at $295 \mathrm{~K}$ and $108 \mathrm{~K}$. (b) Top view of the finite element model with stress distribution along the type I cantilever. (c) Schematic drawing of a bent cantilever at low temperature. (d) Micrographs of uncoated cantilevers of type III at $295 \mathrm{~K}$ and $108 \mathrm{~K}$. (e) same as (d) after chemical treatment. 
with ANSYS 5.7. The geometry of the type I cantilever was modeled with an ANSYS preprocessor unit and meshed in 374 SHELL91 elements. Each element was composed of two layers; the material properties and real constants which correspond to those of $\mathrm{Si}_{3} \mathrm{~N}_{4}$ and gold, respectively. The thicknesses of the layers were set to $400 \mathrm{~nm}$ for $\mathrm{Si}_{3} \mathrm{~N}_{4}$ and $10 \mathrm{~nm}$ for the gold coating. The boundary conditions consisted of blocking all the degrees of freedom of the nodes located at the base of the cantilever and by imposing a temperature change of $180 \mathrm{~K}$ over all of the nodes. The result of this analysis is shown in Fig. 3(b), where bending of $15^{\circ}$ is presented for the case of cantilevers coated on one side. Our estimation from micrographs acquired using cryoSEM [Fig. $3(\mathrm{a})]$ shows bending of $6^{\circ}$. This value is considerably lower since the cantilever in that case was coated on both sides (especially to reduce bending). The gold chromium thickness of the coating on the tip side of the cantilever is declared by the producer to be $40 \mathrm{~nm}$. With the measured bending, we can conclude that the reflective side of cantilever has a layer approximately $5 \mathrm{~nm}$ thicker. This has dramatic consequences for low temperature applications.

Type III cantilevers were investigated before [Fig. 3(d)] and after [Fig. 3(e)] functionalization, which renders them hydrophobic. Biological samples generally have a hydrophilic surface and, when scanned by a hydrophilic tip like the ones commercially available, the tip will adhere to it. Furthermore, they accommodate a layer of water covering their surface even when scanned under a controlled atmosphere. To compensate for its adhesion to the molecule surface, the tip must be pulled away, increasing the load on the cantilever. This leads to an unstable situation and a loss of resolution. This kind of problem has already been addressed in literature. ${ }^{20}$ Knapp et al. ${ }^{21}$ suggested the use of a tip which was previously rendered hydrophobic for contact mode AFM in a humid atmosphere. They found a net improvement in resolution when imaging biomolecules with hydrophobic tips.

The hydrophobic cantilever/tips used for the current study were made in the following manner: Commercially available type III cantilevers were cleaned with an aqueous soap (microclean detergent) solution and then rinsed several times with ultrapure bidistilled filtered water. After drying, they were placed into a special chamber for a reaction of the $\mathrm{Si}_{3} \mathrm{~N}_{4}$ surface with $1 \mathrm{H}, 1 \mathrm{H}, 2 \mathrm{H}, 2 \mathrm{H}$ - Perfluorodecyltrichlorosilane $(\mathrm{ABCR} \mathrm{GmbH})$ under water-free conditions at a temperature of $130^{\circ} \mathrm{C}$ for 1.0 to $1.5 \mathrm{~h}$. After completion of the reaction, the cantilevers were then removed from the chamber and stored in fluoroware containers until used.

As shown in Fig. 3(e), no bending was observed with uncoated cantilevers (type III). This was also the case even after functionalization.

Since the type I cantilevers bend when cooled, a tipless part of the cantilever touches the sample during approach before the tip. This is schematized in Fig. 3(c). Most probably, this explains why we were not able to obtain good quality AFM images with these cantilevers below $200 \mathrm{~K}$. We can, therefore, conclude that this type of cantilever is not recommended for low-temperature measurements.

\section{CONCLUSION}

AFM probes for low-temperature applications were characterized. Three types of contact mode triangular cantilevers were studied at low temperatures. The measurement of resonant frequency spectra allowed us to calculate spring constants. A temperature dependent shift of the peak frequency is observed. Force-distance curves acquired at low temperature show that the hydrophobicity of both the tip and sample is appropriate. Micrographs of coated cantilevers recorded using scanning electron microcopy at low temperature confirm its bending due to a slight difference in the thickness of the metal coating of the cantilever. On the other hand, chemical treatment of uncoated cantilevers does not cause bending at low temperatures and was confirmed to be suitable for lowering adhesion forces and improving the quality of AFM images at low temperatures.

\section{ACKNOWLEDGMENTS}

The authors would like to thank Pierre A. Standelmann and Ollivier Pujol from ICEM, EPFL in Lausanne for providing scanning electron microscopy measurements. This work was partially funded by the Swiss National Science Foundation under Grant Nos. 2000-57133.99, 2000065160.00, and 3100-052587.97.

${ }^{1}$ G. Binning, C. F. Quate, and C. Gerder, Phys. Rev. Lett. 56, 930 (1986).

${ }^{2}$ J. Rychen, T. Ihn, P. Studerus, A. Herrmann, and K. Ensslin, Rev. Sci. Instrum. 70, 2765 (1999).

${ }^{3}$ S. Hembacher, F. J. Giessibl, and J. Mannhart, Appl. Surf. Sci. 188, 445 (2002).

${ }^{4}$ T. R. Albrecht, S. Akamine, T. E. Carver, and C. F. Quate, J. Vac. Sci. Technol. A 8, 3386 (1990).

${ }^{5}$ S. Alexander, L. Hellemans, O. Marti, J. Schneir, V. Elings, P. K. Hansma, M. Longmire, and J. Gurley, J. Appl. Phys. 65, 164 (1989).

${ }^{6}$ A. Radenovic, E. Bystrenová, L. Libioulle, M. Taborelli, J. A. Derose, and G. Dietler, Rev. Sci. Instrum. 74, 1022 (2003).

${ }^{7}$ J. E. Sader, I. Larson, P. Mulvaney, and L. R. White, Rev. Sci. Instrum. 66, 4583 (1995).

${ }^{8}$ J. P. Cleveland, S. Manne, D. Bocek, and P. K. Hansma, Rev. Sci. Instrum. 64, 403 (1993).

${ }^{9}$ J. L. Hutter and J. Bechhoefer, Rev. Sci. Instrum. 64, 1868 (1993).

${ }^{10}$ J. B. Wachtman, W. E. Tefft, D. G. Lam, and C. S. Apstein, Phys. Rev. 122, 1754 (1961).

${ }^{11}$ S. Morita, R. Wiesendanger, and E. Meyer, Noncontact Atomic Force Microscopy (Springer, Berlin, 2002), p. 38.

${ }^{12}$ U. Rabe, K. Janser, and W. Arnold, Rev. Sci. Instrum. 67, 3281 (1996).

${ }^{13}$ N. Kawanishi, H. K. Christenson, and B. W. Ninham, J. Phys. Chem. 94, 4611 (1990).

${ }^{14}$ F. F. Abraham and I. P. Batra, Surf. Sci. 209, L152 (1989).

${ }^{15}$ A. L. Weisenhorn, P. K. Hansma, T. R. Albrecht, and C. F. Quate, Appl. Phys. Lett. 54, 2651 (1989).

${ }^{16}$ J. Yang and Z. Shao, Ultramicroscopy 50, 157 (1993).

${ }^{17}$ B. Cappella and G. Dietler, Surf. Sci. Rep. 34, 1 (1999).

${ }^{18}$ V. F. Petrenko, J. Phys. Chem. B 101, 6276 (1997).

${ }^{19}$ U. Raviv, P. Laurat, and J. Klein, Nature (London) 413, 51 (2001).

${ }^{20}$ H. K. Knapp and A. Stemmer, Surf. Interface Anal. 27, 324 (1999).

${ }^{21}$ H. K. Knapp, R. Guckenberger, and A. Stemmer, Probe Microsc. 1, 247 (1998). 